\title{
Atom-photon, atom-atom and photon-photon entanglement preparation by fractional adiabatic passage
}

\author{
M. Amniat-Talab ${ }^{1,2}$ * S. Guérin ${ }^{1}$ I N. Sangouard ${ }^{1}$, and H.R. Jauslin ${ }^{1}$ \\ ${ }^{1}$ Laboratoire de Physique, UMR CNRS 5027, Université de Bourgogne, B.P. 47870, F-21078 Dijon, France. \\ ${ }^{2}$ Physics Department, Faculty of Sciences, Urmia University, P.B. 165, Urmia, Iran.
}

(Dated: October 9, 2018)

\begin{abstract}
We propose a relatively robust scheme to generate maximally entangled states of (i) an atom and a cavity photon, (ii) two atoms in their ground states, and (iii) two photons in two spatially separate high-Q cavities. It is based on the interaction via fractional adiabatic passage of a three-level atom traveling through a cavity mode and a laser beam. The presence of optical phases is emphasized.

PACS numbers: 42.50.Dv, 03.65.Ud, 03.67.Mn
\end{abstract}

\section{INTRODUCTION}

One of the non-classical aspects of a quantum system made of $N$ parts is entanglement, for which the state vector of the system cannot be written, in any basis, as a tensor product of independent substates. The generation and the controlled manipulation of entangled states of $N$-particle systems is fundamental for the study of basic aspects of quantum theory [1, 2]. The idea is to apply a set of controlled coherent interactions to the particles (atoms, ions, photons) of the system in order to bring them into a tailored entangled state. The physics of entanglement provides the basis of applications such as quantum information processing and quantum communications. Very recently teleportation of quantum states has been realized [3, 4] using atom-atom entanglement following the proposal of Bennett et al. [5]. Particles can then be viewed as carriers of quantum bits of information and the realization of engineered entanglement is an essential ingredient of the implementation of quantum gates [6].

Most experimental realizations of entanglement have been implemented with photons. Although the individual polarization states of photons are easily controlled, and their quantum coherence can be preserved over many kilometers of an optical fiber [7], photons cannot be stored for long times, and manipulations of collective entangled states present considerable difficulties even when photons are confined in the same cavity. The creation of long lived entangled pairs with atoms, on the other hand, is a relatively recent pursuit which may provide reliable quantum information storage. The entangled state of a pair of two-level atoms using pulse area technique in a microwave cavity has been realized by Hagley et al. [8] based on the proposal of Cirac and Zoller [9]. However the pulse area technique is not robust with respect to the velocity of the atoms and the exact-resonance condition. Recently a different scheme has been proposed [10] to entangle two atoms using a tripod STIRAP technique in a four-level atom-cavity-laser system in which one of the pulses corresponds to the field of a cavity mode. Manipulation of entanglement of two atoms in

\footnotetext{
*Electronic address: amniyatm@u-bourgogne.fr
}

†Electronic address: sguerin@u-bourgogne.fr this scheme, however, requires to control a geometric phase via an integral of Hamiltonian parameters over a closed path in parameter space which is difficult in experimental implementations. The generation of atom-photon entanglement has also been proposed in [11] in a tripod-like laser-atom-cavity system which sustains two cavity modes.

In $\Lambda$-type systems, fractional STIRAP (f-STIRAP) is a variation of STIRAP [12] which allows the creation of any preselected coherent superposition of the two degenerate ground states [13]. As in STIRAP, the Stokes pulse linking the initially unpopulated states $|e\rangle$ and $\left|g_{2}\right\rangle$, arrives before the pump pulse linking the initially populated state $\left|g_{1}\right\rangle$ to the excited state $|e\rangle$, but unlike STIRAP where the Stokes pulse vanishes first, here the two pulses vanish simultaneously while maintaining a constant finite ratio of amplitudes. The f-STIRAP has been shown to increase the coherence between the lower states of $\Lambda$-systems in nonlinear optics experiments [14]. The advantage of STIRAP is the robustness of its control with respect to the precise tuning of pulse areas, pulse delay, pulse widths, pulse shapes, and detunings. Since f-STIRAP requires a precise ratio of pulse endings, it is not as robust as STIRAP if two different pulses are used. However in specific circumstances where a laser of elliptic polarization can be used, fSTIRAP can be made as robust as STIRAP [13]. In f-STIRAP as in STIRAP, if the evolution is adiabatic (for instance with a slow transit of atoms across cw fields), the dynamics of the system follows an adiabatic dark state which does not involve the excited atomic state $|e\rangle$. Therefore this technique is immune to the detrimental consequences of atomic spontaneous emission. The STIRAP technique has interesting applications in the generation of coherent superposition of Fock states [15, 16] and of maximally polarization-entangled photon states [17] in an optical cavity.

In this paper we consider neutral three-level $\Lambda$-type atoms with two-fold degenerate ground states $\left|g_{1}\right\rangle,\left|g_{2}\right\rangle$ and an excited state $|e\rangle$. The qubits are stored in the two ground states of the atoms. Our scheme to create the entangled states is based on the resonant interaction of the atoms with an optical cavity mode and a laser field as follows:

(i) Atom-photon entanglement: the first atom initially in the ground state $\left|g_{1}\right\rangle$ interacts with the cavity mode (initially in the vacuum state) and the laser field in the frame of f-STIRAP with the cavity-laser sequence (meaning that the atom meets 


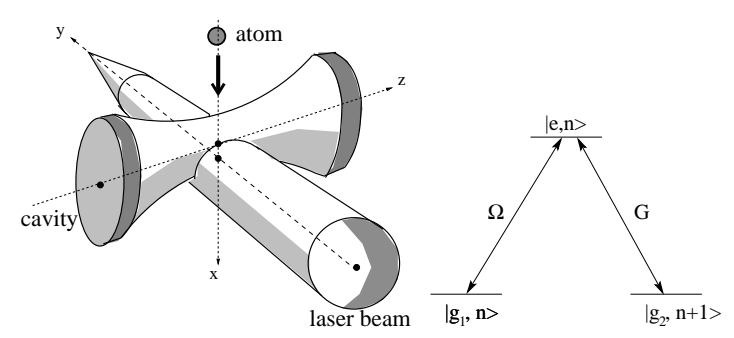

FIG. 1: Experimental configuration and the linkage pattern of atomcavity-laser system with a two-photon resonance between states $\left|g_{1}, n\right\rangle$ and $\left|g_{2}, n+1\right\rangle$.

first the cavity).

(ii) Atom-atom entanglement: when the first atom has left the interaction region, the second atom initially in the ground state $\left|g_{2}\right\rangle$ interacts with the laser-cavity sequence in the frame of STIRAP. After the creation of an entangled state of the atoms, the cavity mode is left in the vacuum state which is not entangled with the two atoms. Therefore the decoherence effect of the cavity damping does not affect the atom-atom entanglement before and after the interaction. The cavity damping must be negligible only during the time of entanglement preparation.

(iii) Photon-photon entanglement: after the interaction of the atom with the first cavity and the laser field in the frame of f-STIRAP, the same atom interacts with the same laser field and the second cavity in the frame of STIRAP. At the end of the interaction, the atomic state factorizes and is left in the ground state $\left|g_{2}\right\rangle$.

\section{CONSTRUCTION OF THE EFFECTIVE HAMILTONIAN}

Figure 1 represents the linkage pattern of the atom-cavitylaser system. The laser pulse associated to the Rabi frequency $\Omega(t)$ couples the states $\left|g_{1}\right\rangle$ and $|e\rangle$, and the cavity mode (Stokes pulse) with Rabi frequency $G(t)$ couples the states $|e\rangle$ and $\left|g_{2}\right\rangle$. The Rabi frequencies $\Omega(t)$ and $G(t)$ are chosen real and positive without loss of generality. These two fields interact with the atom with a time delay, each of the fields is in one-photon resonance with the respective transition. The semiclassical Hamiltonian (i.e. with a classical laser field) of this system in the rotating-wave approximation can be written in the atomic basis $\left\{\left|g_{1}\right\rangle,|e\rangle,\left|g_{2}\right\rangle\right\}$ (in units of $\hbar$ ) as

$H(t)=\omega_{C} a^{\dagger} a+\left[\begin{array}{ccc}0 & \Omega(t) e^{i\left(\omega_{L} t+\varphi_{L}\right)} & 0 \\ \Omega(t) e^{-i\left(\omega_{L} t+\varphi_{L}\right)} & \omega_{e} & G(t) a \\ 0 & G(t) a^{\dagger} & 0\end{array}\right]$

where $a\left(a^{\dagger}\right)$ is the annihilation(creation) operator for the cavity mode, $\omega_{e}$ is the energy of the atomic excited state $\left(\omega_{g_{1}}=\right.$ $\left.\omega_{g_{2}}=0\right), \omega_{C}, \omega_{L}$ are the carrier frequencies of the cavity mode and the laser field respectively $\omega_{C}=\omega_{L}=\omega_{e}$, and $\varphi_{L}$ is the initial phase of the laser field. The time-dependence of
$\Omega(t)$ and $G(t)$ comes from the motion of the atom across the laser and cavity fields and the time origin is defined below.

The Hamiltonian $H(t)$ is block-diagonal in the manifolds $\left\{\left|g_{1}, n\right\rangle,|e, n\rangle,\left|g_{2}, n+1\right\rangle ; n=0,1,2, \ldots\right\}$, where $n$ is the number of photons in the cavity mode, $|e, n\rangle \equiv|e\rangle \otimes|n\rangle$ and $|n\rangle$ is a $n$-photon Fock state. The vector $\left|g_{2}, 0\right\rangle$ is not coupled to any other ones, i.e. $\left|g_{2}, 0\right\rangle$ is a stationary state of the system. One can thus restrict the problem to the projection of the Hamiltonian in the subspace $\left\{\left|g_{1}, 0\right\rangle,|e, 0\rangle,\left|g_{2}, 1\right\rangle\right\}$ :

$$
\begin{aligned}
H_{P} & :=P H P \\
P & =\left|g_{1}, 0\right\rangle\left\langle g_{1}, 0|+| e, 0\right\rangle\left\langle e, 0|+| g_{2}, 1\right\rangle\left\langle g_{2}, 1\right|
\end{aligned}
$$

if one considers the initial state $\left|g_{1}, 0\right\rangle$. The associated dynamics is determined by the Schrödinger equation $i \frac{\partial}{\partial t}|\Psi(t)\rangle=$ $H_{P}(t)|\Psi(t)\rangle$. The effective Hamiltonian can be written as

$$
\begin{aligned}
H^{\mathrm{eff}} & =R^{\dagger} H_{P} R-i R^{\dagger} \frac{\partial R}{\partial t}, \\
R(t) & =\left|g_{1}, 0\right\rangle\left\langle g_{1}, 0\right|+e^{-i \omega_{L} t}\left(|e, 0\rangle\left\langle e, 0|+| g_{2}, 1\right\rangle\left\langle g_{2}, 1\right|\right),
\end{aligned}
$$

which reads in the basis $\left\{\left|g_{1}, 0\right\rangle,|e, 0\rangle,\left|g_{2}, 1\right\rangle\right\}$

$$
H^{\mathrm{eff}}(t)=\left[\begin{array}{ccc}
0 & \Omega(t) e^{i \varphi_{L}} & 0 \\
\Omega(t) e^{-i \varphi_{L}} & 0 & G(t) \\
0 & G(t) & 0
\end{array}\right]
$$

with the corresponding dynamics $i \frac{\partial}{\partial t}|\Phi(t)\rangle=H^{\mathrm{eff}}(t)|\Phi(t)\rangle$. The relation between $|\Psi\rangle$ and $|\Phi\rangle$ is established by unitary transformation $R$ as $|\Psi\rangle=R|\Phi\rangle$.

\section{ATOM-PHOTON ENTANGLEMENT}

The system is taken to be initially in the state $\left|g_{1}, 0\right\rangle$,

$$
|\Phi(-\infty)\rangle=\left|g_{1}, 0\right\rangle=|\Psi(-\infty)\rangle
$$

and we will transform it at the end of interaction into the atomphoton entangled state

$$
\begin{aligned}
& |\Phi(t \rightarrow+\infty)\rangle=\cos \vartheta\left|g_{1}, 0\right\rangle-e^{-i \varphi_{L}} \sin \vartheta\left|g_{2}, 1\right\rangle \\
& |\Psi(t \rightarrow+\infty)\rangle=\cos \vartheta\left|g_{1}, 0\right\rangle-e^{-i\left(\omega_{L} t+\varphi_{L}\right)} \sin \vartheta\left|g_{2}, 1\right\rangle
\end{aligned}
$$

where $\vartheta$ is a constant mixing angle $(0 \leq \vartheta \leq \pi / 2)$. It is important to notice the presence of the generally unknown absolute phase $\omega_{L} t+\varphi_{L}$ in the resulting entangled state 6b. This optical phase factor was not taken into account by Parkins et al. in the generation of an arbitrary superpositions of Fock states [15]. This phase that changes rapidly as a function of , the time, is expected to be uncontrollable in practice.

One of the instantaneous eigenstates (the dark state) of $H^{\text {eff }}(t)$ which corresponds to a zero eigenvalue and therefore to a zero dynamical phase, is

$$
|D(t)\rangle=\frac{1}{\sqrt{\Omega^{2}(t)+G^{2}(t)}}\left[G(t)\left|g_{1}, 0\right\rangle-\Omega(t) e^{-i \varphi_{L}}\left|g_{2}, 1\right\rangle\right] .
$$




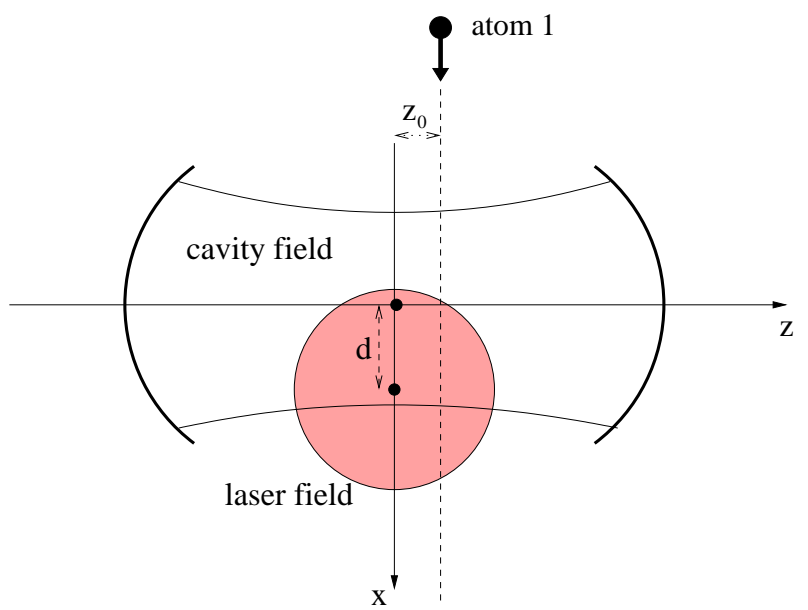

FIG. 2: The geometry of the cavity-mode and the laser fields in the $x z$ plane with different waists $\left(W_{C}>W_{L}\right)$, and the trajectory of the first atom. The specific values of $z_{0}$ and $d$ are chosen such that the atom interacts with the fields via f-STIRAP with the sequence cavity-laser.

As in f-STIRAP, the cavity-mode pulse comes first and is followed after a certain time delay by the laser pulse, but the two pulses vanish simultaneously, that can be asymptotically formulated as

$$
\lim _{t \rightarrow-\infty} \frac{\Omega(t)}{G(t)}=0, \quad \lim _{t \rightarrow+\infty} \frac{\Omega(t)}{G(t)}=\tan \vartheta .
$$

The dark state (7) has consequently the limits $|D(-\infty)\rangle=$ $\left|g_{1}, 0\right\rangle$ and $|D(+\infty)\rangle=\cos \vartheta\left|g_{1}, 0\right\rangle-e^{-i \varphi_{L}} \sin \vartheta\left|g_{2}, 1\right\rangle$ with such a pulse sequence and allows thus one to generate a coherent superposition of states by adiabatic passage. It should be emphasized that this formulation in terms of asymptotics (8) does not describe correctly what occurs at the beginning and the ending of f-STIRAP for a concrete realization which is not strictly adiabatic. In particular, using this asymptotics would give a failure of f-STIRAP for Gaussian pulses (considered below), which do not asymptotically give a constant ending ratio for any delay and pulse width. The inspection of the nonadiabatic couplings [13] shows that what matters is that the Rabi frequency amplitudes end in a constant ratio in a time interval where they are non negligible, and Eq. (8) has to be understood in this sense. The goal in the following is to show that such a pulse sequence can be designed in a cavity by an appropriate choice of the parameters.

In an optical cavity, the spatial variation of the atom-field coupling for a Hermite-Gauss TEM ${ }_{m n}$ mode is given by

$$
\begin{aligned}
G_{m n}(x, y, z) & =G_{0} H_{m}\left(\frac{\sqrt{2} x}{W_{C}}\right) H_{n}\left(\frac{\sqrt{2} y}{W_{C}}\right) \\
& \times e^{-\left(x^{2}+y^{2}\right) / W_{C}^{2}} \cos \left(\frac{2 \pi z}{\lambda}\right),
\end{aligned}
$$

where $G_{0}=\mu \sqrt{\omega_{C} /\left(2 \epsilon_{0} V_{\text {mode }}\right)}$ with $\mu, V_{\text {mode }}$ respectively the dipole moment of the atomic transition and the effective volume of the cavity mode. The transverse distribution is determined by Hermite polynomials $H_{m}, H_{n}$ and the cavity waist $W_{C}$ [18]. The standing wave along the cavity $\mathrm{z}$-axis gives rise to a $\cos (2 \pi z / \lambda)$ dependence of the mode with the wavelength $\lambda$. A particular transverse mode is selected by adjusting the cavity length. We consider the maximum coupling mode $\mathrm{TEM}_{00}$ resonant with the $|e\rangle \leftrightarrow\left|g_{2}\right\rangle$ transition of the atom

$$
G(x, y, z)=G_{0} e^{-\left(x^{2}+y^{2}\right) / W_{C}^{2}} \cos \left(\frac{2 \pi z}{\lambda}\right) .
$$

Figure 2 shows a situation where an atom initially in the state $\left|g_{1}\right\rangle$ falls with velocity $v$ (on the $y=0$ plane and $z=z_{0}$ line) through an optical cavity initially in the vacuum state $|0\rangle$ and then encounters the laser beam, which is parallel to the $y$ axis (orthogonal to the cavity axis and the trajectory of the atom). The laser beam of waist $W_{L}$ is resonant with the $|e\rangle \leftrightarrow\left|g_{1}\right\rangle$ transition. The distance between center of the cavity and the laser axis is $d$. The traveling atom encounters the time dependent and delayed Rabi frequencies of the cavity and the laser fields as follows

$$
\begin{aligned}
& G(t)=G_{0} e^{-(v t)^{2} / W_{C}^{2}} \cos \left(\frac{2 \pi z_{0}}{\lambda}\right), \\
& \Omega(t)=\Omega_{0} e^{-z_{0}^{2} / W_{L}^{2}} e^{-(v t-d)^{2} / W_{L}^{2}},
\end{aligned}
$$

where the time origin is defined when the atom meets the center of the cavity $x=0$. The appropriate values of $z_{0}$ and $d$ that lead to the f-STIRAP process can be extracted from a contour plot of the final population $P_{\left|g_{1}, 0\right\rangle}:=\left|\left\langle g_{1}, 0 \mid \Phi(+\infty)\right\rangle\right|^{2}$ as a function of $z_{0}$ and $d$ that we calculate numerically (see Fig. 3). The white dot in Fig. 3 shows values of $z_{0}$ and $d$ to obtain a f-STIRAP process with $\vartheta \simeq \pi / 4$ (called halfSTIRAP). It has been chosen such that at the end of interaction $P_{\left|g_{1}, 0\right\rangle} \simeq P_{\left|g_{2}, 1\right\rangle} \simeq 0.5$ and $P_{|e, 0\rangle} \simeq 0$.

Figure 4 shows (a) the cavity-laser pulse sequence of halfSTIRAP for the first atom, and (b) the time evolution of populations which shows half-half population for the states $\left|g_{1}, 0\right\rangle,\left|g_{2}, 1\right\rangle$ and zero population for the state $|e, 0\rangle$ at the end of the interaction. This case corresponds to the generation of the maximally atom-photon entangled state $1 / \sqrt{2}\left(\left|g_{1}, 0\right\rangle-\right.$ $\left.e^{-i\left(\omega_{L} t+\varphi_{L}\right)}\left|g_{2}, 1\right\rangle\right)$ by adiabatic passage. Assuming Gaussian pulse profiles for $\Omega(t)$ and $G(t)$ of widths $T_{L}=W_{L} / v$ and $T_{C}=W_{C} / v$ respectively, we have the sufficient condition of adiabaticity [13]:

$$
\Omega_{0} T_{L}, G_{0} T_{C} \gg 1
$$

We remark that the case $\vartheta=\pi / 2$ corresponds to the standard STIRAP with the final state $|\Psi(t \rightarrow+\infty)\rangle=$ $-e^{-i\left(\omega_{L} t+\varphi_{L}\right)}\left|g_{2}, 1\right\rangle$, i.e. to the generation of a single-photon Fock state in the cavity mode without population transfer to the atomic excited state at the end of interaction. Here the optical phase factor appears as an irrelevant global phase factor. A one-photon Fock state has been produced in such a way in an optical cavity via STIRAP by Hennrich et al. [19] based on the proposal of Refs. [15, 20]. A robust scheme for the generation of multi-photon Fock states in a microwave cavity via bichromatic adiabatic passage has been proposed in Ref. [21]. 

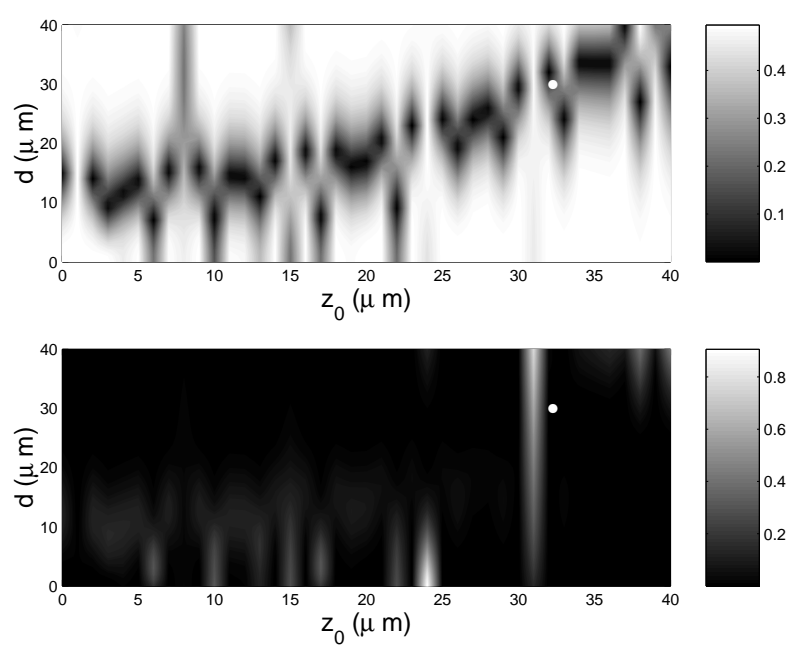

FIG. 3: Top panel: contour plot of the final population $\left|\frac{1}{2}-P_{\left|g_{1}, 0\right\rangle}\right|$ as a function of $z_{0}$ and $d$ (black areas correspond to approximately half population transfer) with the pulse parameters as $W_{L}=20 \mu \mathrm{m}$, $W_{C}=30 \mu \mathrm{m}, v=2 \mathrm{~m} / \mathrm{s}, \lambda=780 \mathrm{~nm}, \quad \Omega_{0}=50 \frac{v}{W_{L}}, G_{0}=$ $50 \frac{v}{W_{C}}$. Bottom panel: the same plot for the population of the excited state $P_{|e, 0\rangle}:=|\langle e, 0 \mid \Phi(+\infty)\rangle|^{2}$ where black areas correspond to approximately zero population transfer. The white dot shows specific values of $z_{0}$ and $d$ used in Fig. 4 to obtain a half-STIRAP process.

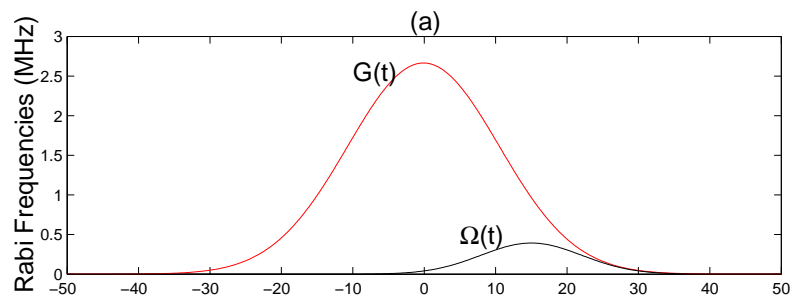

(b)

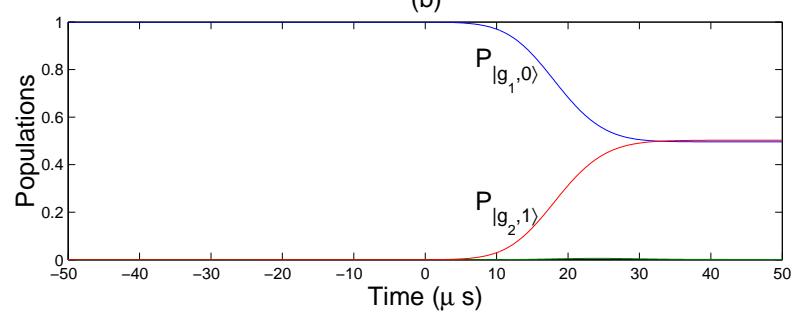

FIG. 4: (a) Rabi frequencies of the cavity-mode and the laser field for the first atom corresponding to the same pulse parameters and the specific values $z_{0}=31.9 \mu \mathrm{m}, d=30.2 \mu \mathrm{m}$ of the white dot in Fig. 3 (b) Time evolution of the populations for the trajectory of the first atom which represents a half-STIRAP.

\section{ATOM-ATOM ENTANGLEMENT}

In this section we consider a situation where the first atom has been entangled with the cavity mode via f-STIRAP as described by Eq. (6b), and the second atom initially in the ground state $\left|g_{2}^{(2)}\right\rangle$ is going to interact with the same laser and cavity-mode fields but through a STIRAP process (see Fig. 5). The superscript labels the two atoms. The state of the atom ${ }^{(2)}$ -

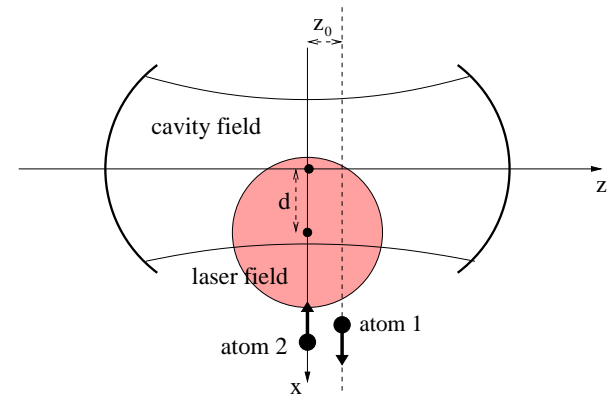

FIG. 5: The proposed geometry of the cavity and the laser fields in $x z$ plane as well as the trajectory of the atoms for generation of atom-atom entanglement. The second atom initially in the ground state $\left|g_{2}\right\rangle$ arrives at the center of the cavity with a time delay $\tau$. This atom encounters the sequence laser-cavity on the line $z=0$.

atom $^{(1)}$-cavity system after entanglement of the atom ${ }^{(1)}$ reads

$|\Psi(t)\rangle=\left|g_{2}^{(2)}\right\rangle\left(\cos \vartheta\left|g_{1}^{(1)}, 0\right\rangle-e^{-i\left(\omega_{L} t+\varphi_{L}\right)} \sin \vartheta\left|g_{2}^{(1)}, 1\right\rangle\right)$

The second atom moves on the line $z=0$ in the same plane (see Fig. 5) as the first one such that the two atoms experience the same optical phase $e^{i \omega t}$ of the laser field. It encounters time-dependent and delayed Rabi frequencies given by

$$
\begin{aligned}
& G^{(2)}(t)=G_{0} e^{-[v(t-\tau)]^{2} / W_{C}^{2}}, \\
& \Omega^{(2)}(t)=\Omega_{0} e^{-[v(t-\tau)+d]^{2} / W_{L}^{2}},
\end{aligned}
$$

where $\tau$ is the time delay between the two atoms. By standard STIRAP, with the sequence of laser-cavity (see Fig. $6)$, we can transfer the population from the initial state $\left|g_{2}^{(2)}, 1\right\rangle$ to the final state $\left|g_{1}^{(2)}, 0\right\rangle$. On the other hand the state $\left|g_{2}^{(2)}, 0\right\rangle$ is stationary with respect to this STIRAP process. Using the transformation $R^{(2)}(t)=\left|g_{1}^{(2)}, 0\right\rangle\left\langle g_{1}^{(2)}, 0\right|+$ $e^{-i \omega_{L} t}\left(\left|e^{(2)}, 0\right\rangle\left\langle e^{(2)}, 0|+| g_{2}^{(2)}, 1\right\rangle\left\langle g_{2}^{(2)}, 1\right|\right)$, this results in

$$
\left|g_{2}^{(2)}, 0\right\rangle \rightarrow\left|g_{2}^{(2)}, 0\right\rangle, \quad-e^{-i\left(\omega_{L} t+\varphi_{L}\right)}\left|g_{2}^{(2)}, 1\right\rangle \rightarrow\left|g_{1}^{(2)}, 0\right\rangle \text {. }
$$

Hence, if the second atom encounters the laser field before the cavity field in the frame of a standard STIRAP, the final state of the atom $^{(2)}$-atom ${ }^{(1)}$-cavity system will be

$$
\begin{aligned}
|\Psi(+\infty)\rangle & =\cos \vartheta\left|g_{2}^{(2)}, 0\right\rangle\left|g_{1}^{(1)}\right\rangle+\sin \vartheta\left|g_{1}^{(2)}, 0\right\rangle\left|g_{2}^{(1)}\right\rangle \\
& =|0\rangle\left(\cos \vartheta\left|g_{2}^{(2)}\right\rangle\left|g_{1}^{(1)}\right\rangle+\sin \vartheta\left|g_{1}^{(2)}\right\rangle\left|g_{2}^{(1)}\right\rangle\right)
\end{aligned}
$$

Since the cavity-mode state factorizes and is left in the vacuum state, there is no projection noise when one traces over the unobserved cavity field, and the cavity is ready to prepare another entanglement. We can manipulate this entanglement coherently to reach the maximal atom-atom entanglement by tuning the ratio of fields such that $\tan \vartheta=1$ in the f-STIRAP stage, as shown in Figs. 3 and 4.

Figure 6 shows the successful STIRAP process for the second atom (moving along the line $z=0$ ) that allows one to generate the entangled state 16 . 

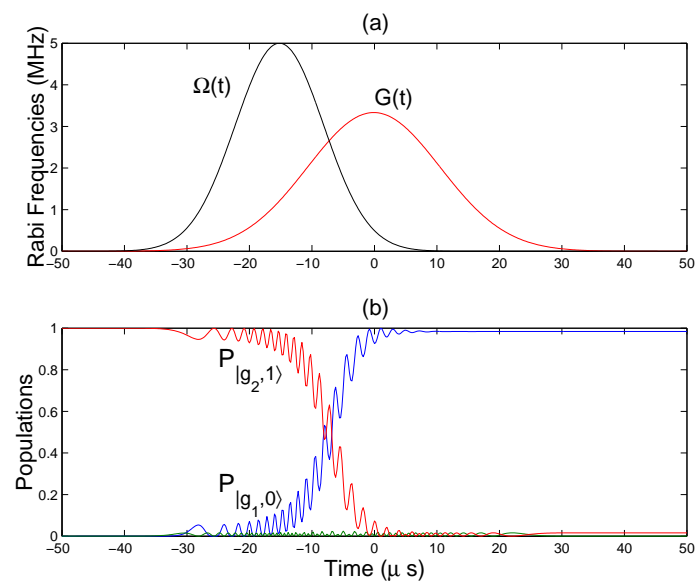

FIG. 6: (a) Rabi frequencies of the fields for the second atom travelling on the line $z=0$ with the same parameters of Fig. 4 (b) Time evolution of the populations.

The generation of atom-atom entanglement with the two atoms interacting simultaneously ( $\tau=0$ in Fig. 5) with the cavity mode, that can be described by a two-atom dark state presented in Ref. [22], will be discussed elsewhere.

\section{PHOTON-PHOTON ENTANGLEMENT}

In Refs. [23, 24, 25] among many others, different schemes have been proposed to entangle two and three microwave cavities through the interaction with a Rydberg atom. A method of generating particular entangled states of two cavities appeared as an intermediate step in the teleportation procedure proposed by Davidovich et al. [26]. Here we propose another scheme to entangle two optical cavities interacting with an atom. We consider a situation where an atom has been entangled with the first single-mode cavity via a f-STIRAP technique as described in section II and it interacts next with another single-mode optical cavity, initially in the vacuum state, and the same laser field (see Fig. 77). The distance $z_{0}$ between the axis of motion of the atom and the center of the first cavity, ensures having an f-STIRAP process for the first cavity. Hence the state of the cavity ${ }^{(2)}$-atom-cavity ${ }^{(1)}$ system after the f-STIRAP process is

$|\Psi(t)\rangle=\left|0^{(2)}\right\rangle\left(\cos \vartheta\left|g_{1}, 0^{(1)}\right\rangle-e^{-i\left(\omega_{L} t+\varphi_{L}\right)} \sin \vartheta\left|g_{2}, 1^{(1)}\right\rangle\right)$

where the superscripts denote the number of cavities. If the atom interacts with the second cavity in the frame of a standard STIRAP and the atom encounters the cavity mode before the laser pulse, since the state $\left|g_{1}, 0^{(2)}\right\rangle$ evolves to $\left|g_{2}, 1^{(2)}\right\rangle$ and the state $\left|g_{2}, 0^{(2)}\right\rangle$ does not change during the interaction:

$$
\begin{aligned}
\left|g_{1}, 0^{(2)}\right\rangle & \rightarrow-e^{-i\left(\omega_{L} t+\varphi_{L}\right)}\left|g_{2}, 1^{(2)}\right\rangle \\
\left|g_{2}, 0^{(2)}\right\rangle & \rightarrow\left|g_{2}, 0^{(2)}\right\rangle
\end{aligned}
$$

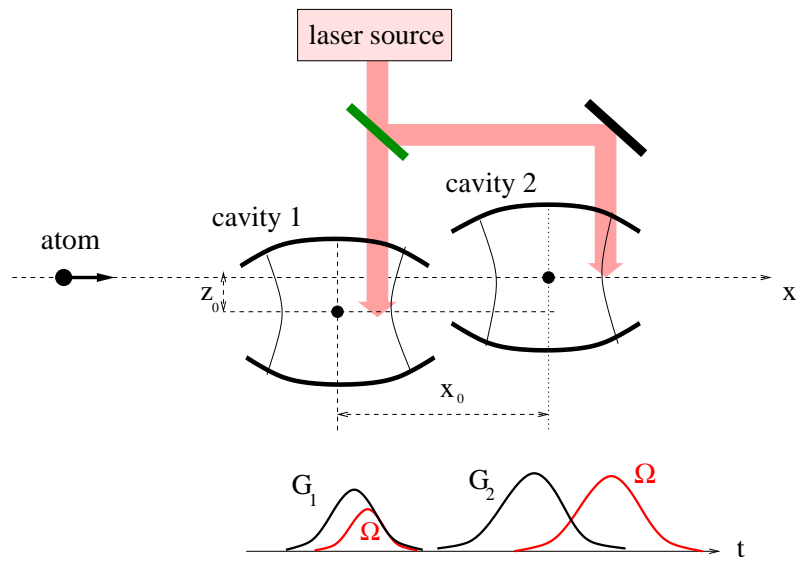

FIG. 7: Proposed setup for entangling of two photons generated in two optical cavities interacting with a three-level atom and a laser field, and the schematic pulse sequences. The atom interacts with the first cavity via f-STIRAP in the same conditions of Fig. 2] and next with the second cavity via standard STIRAP with the sequence cavity-laser in each one. The propagation direction of the laser beams are perpendicular to the plane $\mathrm{xz}$ as the figure 1.

the final state of the system will be (up to an irrelevant common phase factor)

$$
|\Psi(+\infty)\rangle=\left|g_{2}\right\rangle\left(\cos \vartheta\left|1^{(2)}, 0^{(1)}\right\rangle+e^{i \alpha} \sin \vartheta\left|0^{(2)}, 1^{(1)}\right\rangle\right)
$$

where $\alpha=2 \pi\left(x_{0}^{2}+z_{0}^{2}\right)^{1 / 2} / \lambda$ is the phase shift of the laser field due to the optical path difference between the two cavities. Since the atomic state factorizes and is left in the ground state $\left|g_{2}\right\rangle$, the atom does not have spontaneous emission and it could be used to prepare another entanglement.

\section{DISCUSSIONS AND CONCLUSIONS}

We have proposed a robust scheme to generate atomphoton, atom-atom and photon-photon entanglement, using a combination of f-STIRAP and STIRAP techniques in $\Lambda$ systems. This scheme is robust with respect to variations of the velocity of the atom $v$, of the peak Rabi frequencies $G_{0}, \Omega_{0}$ and of the pulse detunings, but not with respect to the parameters $d, z_{0}$. For given values of $W_{C}, W_{L}$, the adapted values of $d$ and $z_{0}$ in the f-STIRAP process can be determined from a contour plot of the final populations as explained in section III

The presence of optical phases in the case of atom-photon entanglement, expected to be uncontrollable, was emphasized. In the case of atom-atom entanglement the optical phase is not present, as long as the two atoms move in the same plane perpendicular to the propagation direction of the laser beam.

Dissipation in the form of spontaneous emission and cavity damping is another important practical issue. The adiabatic passage technique is robust against the effects of spontaneous emission, as the excited atomic state is never appreciably populated. Cavity damping is certainly a problem as its effects 
come into play as soon as the cavity mode is excited, leading to a degradation of the adiabatic transfer. In this analysis we have assumed that the interaction time between the atom and the fields $T_{\mathrm{int}} \approx W_{C} / v \approx W_{L} / v$ is short compared to the cavity lifetime $T_{\text {cav }}$, which are essential for an experimental realization.

Since the decay rate of the cavity scales with the number of photons present in the cavity $\left(T_{\text {int }} \ll T_{\text {cav }} / n\right)$, our scheme involving only one cavity photon requires $T_{\text {int }} \ll T_{\text {cav }}$. In a real experiment, it is desirable that the entangled states are as long-lived as possible. This requires in the optical domain, where $T_{\text {int }} \approx 15 \mu \mathrm{s}$, a cavity lifetime of $T_{\text {cav }} \gg 15 \mu \mathrm{s}$. This is beyond the currently available optical cavities where $T_{\text {cav }} \approx 1$ $\mu \mathrm{s}$. One could still consider the generation of atom-atom entanglement in an optical cavity using a two-atom dark state of the type presented in Ref. [22] which does not require such a stringent constraint for the cavity lifetime. In the microwave domain, cavities with a photon lifetime of $1 \mathrm{~ms} \mathrm{[27]} \mathrm{and} \mathrm{of} 0.3$ $\mathrm{s}$ [28] have been made. The upper limit of interaction time is $T_{\text {int }}=100 \mu \mathrm{s}$ (atom with a velocity of $100 \mathrm{~m} / \mathrm{s}$ with the cavity mode waist of $W_{C}=6 \mathrm{~mm}$ ). The condition of global adiabaticity $G_{0} T_{\text {int }} \gg 1$ for the typical value of $G_{0} \approx 0.15 \mathrm{MHz}$ [27] is well satisfied $G_{0} T_{\text {int }} \approx 15$. The proposed schemes of entanglement generation could be implemented in a microwave cavity by using a maser field and atomic Rydberg states.

\section{Acknowledgments}

M. A-T. wishes to acknowledge the financial support of the MSRT of Iran and SFERE. We acknowledge support from the Conseil Régional de Bourgogne.
[1] A. Einstein, B. Podolski, and N. Rosen, Phys. Rev. 47, 777 (1935).

[2] J. S. Bell, Physics 1, 195 (1965).

[3] M. Riebe, H. Häffner, C. F. Roos, W. Hänsel, J. Benhelm, G. P. Lancaster, T. W. Körber, C. Becher, F. Schmidt-Kaler, D. F. James, et al., Nature 429, 734 (2004).

[4] M. D. Barrett, J. Chiaverini, T. Schaetz, J. Britton, W. M. Itano, J. D. Jost, E. Knill, C. Langer, D. Leibfried, R. Ozeri, et al., Nature 429, 737 (2004).

[5] C. H. Bennett, G. Brassard, C. Crépeau, R. Jozsa, A. Peres, and W. K. Wootters, Phys. Rev. Lett. 70, 1895 (1993).

[6] A. Ekert and R. Jozsa, Rev. Mod. Phys. 68, 3733 (1997).

[7] W. Tittel, J. Brendel, B. Gisin, T. Herzog, H. Zbinden, and N. Gisin, Phys. Rev. A 57, 3229 (1998).

[8] E. Hagley, X. Maitre, G. Nogues, C. Wunderlich, M. Brune, J. M. Raimond, and S. Haroche, Phys. Rev. Lett. 79, 1 (1997).

[9] J. I. Cirac and P. Zoller, Phys. Rev. A 50, R2799 (1994).

[10] S. Q. Gong, R. Unanyan, and K. Bergmann, Eur. Phys. J. D 19, 257 (2002).

[11] B. Sun, M. S. Chapman, and L. You, Phys. Rev. A 69, 042316 (2004).

[12] N. V. Vitanov, T. Halfmann, B. W. Shore, and K. Bergmann, Annu. Rev. Phys. Chem. 52, 763 (2001).

[13] N. V. Vitanov, K. A. Suominen, and B. W. Shore, J. Phys. B 32, 4535 (1999).

[14] V. A. Sautenkov, C. Y. Ye, Y. V. Rostovtsev, G. R. Welch, and M. O. Scully, quant-ph/0311076 (2003).
[15] A. S. Parkins, P. Marte, P. Zoller, and H. J. Kimble, Phys. Rev. Lett. 71, 3095 (1993).

[16] A. S. Parkins, P. Marte, P. Zoller, O. Carnal, and H. J. Kimble, Phys. Rev. A 51, 1578 (1995).

[17] W. Lange and H. J. Kimble, Phys. Rev. A 61, 063817 (2000).

[18] M. Keller, B. Lange, K. Hayasaka, W. Lange, and H. Walther, J. Phys. B 36, 613 (2003).

[19] M. Hennrich, T. Legero, A. Kuhn, and G. Rempe, Phys. Rev. Lett. 85, 4872 (2000).

[20] A. Kuhn, M. Hennrich, T. Bondo, and G. Rempe, Appl. Phys. B 69, 373 (1999).

[21] M. Amniat-Talab, S. Lagrange, S. Guérin, and H. R. Jauslin, Phys. Rev. A 70, 013807 (2004).

[22] T. Pellizzari, S. A. Gardiner, J. I. Cirac, and P. Zoller, Phys. Rev. Lett. 75, 3788 (2001).

[23] C. C. Gerry, Phys. Rev. A 54, R2529 (1996).

[24] J. A. Bergou and M. Hillery, Phys. Rev. A 55, 4585 (1997).

[25] D. E. Browne and M. B. Plenio, Phys. Rev. A 67, 012325 (2003).

[26] L. Davidovich, N. Zagury, M. Brune, J. M. Raimond, and S. Haroche, Phys. Rev. A 50, R895 (1994).

[27] J. M. Raimond, M. Brune, and S. Haroche, Rev. Mod. Phys. 73, 565 (2001).

[28] S. Brattke, B. T. H. Varcoe, and H. Walther, Phys. Rev. Lett. 86, 3534 (2001). 